W. Jilg, F. Deinhardt

\title{
Vaccination Against Hepatitis B - The Current Situation
}

In 1971, Krugman demonstrated that inoculation of boiled serum obtained from an HBsAg carrier prevented subsequent hepatitis B infection. At that time, as now, all attempts to propagate the virus in cell culture and prepare a vaccine in the conventional way had failed, meaning that Krugman's experiments formed the basis for the development of an $\mathrm{HB}$ vaccine made from the plasma of chronic HBsAG carriers. Hepatitis B vaccines were developed by several groups, and the efficacy, immunogenicity and safety of the vaccines were demonstrated in large clinical studies, such as the famous double-blind trials conducted on New York homosexuals by Szmuness and on French dialysis patients and personnel by Maupas. In 1981, the vaccine was licensed in the U.S.A., and some months later in most European countries. The results of the initial studies have since been confirmed by the vaccination of about three million people to date (for an overview see reference 1).

In a healthy adult population, more than $90 \%$ of the vaccinees develop the protective antibody, anti-HBs, after three vaccinations; protection is achieved with a vaccine efficacy of about $90 \%$. Side-effects are negligible. In none of the cases have hepatitis $B$, hepatitis non-A non-B or the acquired immunodeficiency syndrome (AIDS) been transmitted by the vaccine; the susceptibility of LAV/ HTLV III is such that it would be readily inactivated by each of the inactivation steps used in vaccine preparation. With the exception of allergies to formaldehyde, which is used for inactivation, and to thimerosal, which is added as a bacteriostaticum, there are no contraindications for the use of the $\mathrm{HB}$ vaccine. Gravidity is not a contra-indication; since the HB vaccine is not a live vaccine, there is no risk of embryopathy. On the contrary, the vaccination is strongly indicated for pregnant women at a high risk of contracting hepatitis B, e.g. nurses working in dialysis units or infection wards, because newborns run a high risk of becoming chronic carriers of HBsAg when mothers are HBsAg-positive at the time of birth.

Newborns tolerate the vaccine very well. Although immediate passive immunization with $\mathrm{HB}$ immunoglobulin provides important partial protection for the babies of HBsAg-carrier mothers, passive-active immunization is the most effective measure. The feasibility of such passive-active immunization has been demonstrated in newborns as well as in adults; no interference of the passively transferred immunoglobulins with the developing "active" immunity has been observed. Immediate protection as well as long-term immunity is provided; this is especially important for the newborns of $\mathrm{HBsAg}$-positive mothers, most of whom are chronic carriers and can also infect their children later. Passive-active immunization is also recommended for those who need immediate protection, e.g. persons working in high risk surroundings such as dialysis units and infection wards or persons in contact with HBsAg carriers.

Although the vaccine works well in healthy children and adults, vaccination of immunocompromised persons, especially dialysis patients, is still a problem. These patients are at an increased risk of contracting hepatitis $\mathrm{B}$, and if infected, very often become chronic carriers as a consequence of their impaired immune systems. Protection against hepatitis $B$ is therefore very important for this group; unfortunately, the response to the $\mathrm{HB}$ vaccination is rather poor in these patients. Only 50\%-70\% develop specific antibodies, and the resultant anti-HBs levels are low. Although multiple injections show better results, immune responses are still significantly lower than in healthy persons, and the responses in transplant patients and children with hematologic malignancies are even worse. Despite these less than ideal responses in some groups of individuals, vaccination against $\mathrm{HB}$ is now an accepted preventive measure for people at risk of infection. Some uncertainty, however, still exists about the persistence of anti-HBs after vaccination, and about the need for and the timing of revaccination. Whereas earlier studies lead to the belief that anti-HBs levels would remain higher than $10 \mathrm{IU} / 1$ (considered to be the minimal protective level) for about five years after completion of the vaccination, longterm follow-up studies have shown that in some individuals, anti-HBs levels fall below this value or even disappear much earlier. Recently, a close correlation was demonstrated between the peak anti-HBs concentration after vaccination and the persistence of specific antibodies; thus, a quantitative determination of antiHBs after the basic immunization allows a fairly accurate prediction of the length of protection and consequently of the time for revaccination (2). Whether there is protection below the $10 \mathrm{IU} / \mathrm{ml}$ level is still a matter for debate. In several studies, hepatitis B infections have been observed in vaccinees whose anti-HBs levels had decreased to low (below $50 \mathrm{IU} / 1$ ) or zero. However, in otherwise healthy individuals only subclinical or mild infections have been seen, resulting in most cases in seroconversion to anti-HBc without $\mathrm{HBsAg}$ being present and without liver enzymes being elevated, an argument for some protective effects of the vaccination even when measurable anti-HBs has disappeared (3).

Although the vaccine presently in use is safe and effective, it has the major drawback of being expensive because the costs of the starting material, human plasma, and the extensive purification procedures are high. This seriously limits the use of this vaccine, especially in the

Dr. W. Jilg, Prof. Dr. F. Deinhardt, Max von Pettenkofer Institute, University of Munich, Pettenkoferstr. 9a, D-8000 Munich 2. 
highly endemic areas of Central Africa and Southeast Asia, where the vaccine is needed most. Saving costs by simply reducing the dose of the vaccine to a half or a quarter is possible in children and healthy young adults, but it leads to reduced immunity in older persons. Single intradermal application of a reduced dose, a method which works well with the rabies vaccine, has not been effective with the hepatitis $B$ vaccine. A simplified and cheaper method of preparing the HBs vaccine for plasma has been developed by the Netherlands Red Cross; although the vaccine is highly immunogenic, its production, which includes only one inactivation step by heat, does not meet the recommendations of the WHO regarding purity and inactivation procedures.

After the genome of the HB virus had been cloned and sequenced, production of a vaccine by recombinant DNA technology became possible. HBsAg has been expressed in procaryotic and eucaryotic cells and recently, a vaccine was developed from $\mathrm{HBsAg}$ produced by recombinant techniques in yeast (4). The "yeast HBsAg" is assembled in particles similar to the $22 \mathrm{~nm}$ particles found in human plasma, but it is not glycosylated. Since 1983, this first recombinant human vaccine has been used in clinical trials; about 3,000 volunteers have been immunized so far, and the new vaccine has proven to be as immunogenic and safe as the plasma-derived vaccine (5).

Other techniques are currently being investigated for the production of alternative HB vaccines: the use of synthetic peptides corresponding to HBsAg epitopes (6), the use of anti-idiotypic antibodies (anti-idiotypic antibodies are directed against the antigen binding site of specific antibody and bear the "internal image" of the antigen, in this case $\mathrm{HBsAg}$ ) (7), or the use of recombinant vaccinia virus containing the genomic sequence of $\mathrm{HBsAg}$ as a live vaccine (8). Anti-HBs could be produced in laboratory animals with all three methods, but extensive research is still necessary before vaccines based on these techniques can be used in clinical trials.

Several considerations have arisen in the production of more potent $\mathrm{HB}$ vaccines. Great interest has been raised by the recent observation that the so-called pre-S region of the HBsAg gene codes for two peptides, 55 or 174 amino acids long, which are expressed as the $\mathrm{N}$-terminal portion of the usual 226 amino acid-long HBsAg (9). These compounds have been found to be minor components of the HB virus. They bear the polyalbumin receptor described previously on the surface of $\mathrm{HB}$ virus, and they seem to play a role in the attachment of the virus to the hepatocyte in the first step of infection. In acute hepatitis B, antibodies against these pre-S peptides appear early after the onset of clinical symptoms and may be responsible for the elimination of $\mathrm{HBsAg}$ during convalescence $(10)$. In animals, these peptides were highly immunogenic; inbred strains of mice, incapable of responding to the short 226 amino acid-long $\mathrm{HBsAg}$, after immunization with HBsAg containing the pre-S sequences produce antibodies not only against the pre-S epitopes in a high concentration, but also against the HBsAg determi- nants (11). It has been questioned whether antibodies against the pre-S region could lead to autoimmune phenomena because similarities exist between pre-S and albumin receptors, but so far no evidence of this has been obtained. Preliminary experiments in chimpanzees, previously immunized with isolated pre-S peptides produced by recombinant DNA technology, showed a protective effect of antipre-S antibodies. Vaccines containing the pre-S peptides are currently being developed; clinical tests will establish whether the pre-S determinants show the high immunogenicity they seem to have in laboratory animals.

Finally, it should be remembered that more deaths occurred last year from hepatitis B or its sequelae than from AIDS. In most aspects, the mode of transmission of both diseases is identical, but whereas no prophylaxis for AIDS is available, despite the great investment of research effort and funds, protection against $\mathrm{HB}$ by a vaccine is available. The medical profession should make use of this protection and urge all persons at risk of contracting hepatitis $\mathrm{B}$ to take similar action.

\section{Literature}

1. Stevens, C. E., Taylor, P. E., Tong, M. J., Toy, P. T., Vyas, G. N.: Hepatitis B vaccine. An overview. In: Vyas, G. N., Dienstag, J. L., Hoofnagle, J. H. (eds.): Viral hepatitis and liver disease. Grune \& Stratton, Orlando 1984 , pp. 275-291.

2. Jilg, W., Schmidt, M., Zachoval, R., Deinhardt, F.: Persistenz von Antikörpern gegen Hepatitis B Oberflächenantigen nach Impfung gegen Hepatitis B. Dtsch. Med. Wochenschr. 110 (1985) 205-209.

3. Hadler, S. C., Francis, D. P., Thomson, S., Maynard, J. F., Ostrow, D. G., Braff, E. H., Judson, F. N. and The CDC Multicenter HBV Vaccine Study Group: Longterm efficacy of hepatitis B vaccine. In: Vyas, G. N., Dienstag, J. L., Hoofnagle, J. H. (eds.): Viral hepatitis and liver disease. Grune \& Stratton, Orlando, Florida 1984, p. 693.

4. McAleer, W. J., Buynak, E. B., Maigetter, R. Z., Wampler, D. E., Miller, W. J., Hilleman, M. R.: Human hepatitis B vaccine from recombinant yeast. Nature 307 (1984) 178-180.

5. Brown, S. E., Stanley, C., Howard, C. R., Zuckerman, A. J., Steward, M. W.: Antibody responses to recombinant and plasma derived hepatitis B vaccines. Br. Med. J. 292 (1986) 159-161.

6. Gerin, J. L., Purcell, R. H., Lerner, R. A.: Recombinant DNA and synthetic peptide approaches to HBV vaccine development: immunogenicity and protective efficacy in chimpanzees. In: Chanock, R. M., Lerner, $R$. A. (eds.): Modern approaches to vaccines: molecular and chemical basis of virus virulence and immunogenicity. Cold Spring Harbor Laboratory, New York 1984, pp. 121-125.

7. Kennedy, R. C., Eichberg, J. W., Dreesman, G. R.: Lack of genetic restriction by a potential anti-idiotype vaccine for type $B$ viral hepatitis. Virology 148 (1986) 369-374.

8. Moss, B., Smith, G. L., Gerin, J. L., Purcell, R. H.: Use of vaccinia virus as a vector for the construction of live recombinant hepatitis $B$ virus vaccines. In: Vyas, G. N., Dienstag, J. L., Hoofnagle, J. H. (eds.): Viral hepatitis and liver disease. Grune \& Stratton, Orlando, Florida 1984, pp. 293-305.

9. Heermann, K. H., Goldmann, U., Schwartz, W., Seyffarth, T., Baumgarten, H., Gerlich, W. H.: Large surface proteins of hepatitis B virus containing the pre-S sequence. J. Virol. 52 (1984) 396-402.

10. Budkowska, A., Briantais, M. J., Dubreuil, P., Capel, F., GrangeotKeros, L., Pillot, J.: Detection of antibodies directed against the pre-S gene product of hepatitis B virus: relationship between anti-pre-S response and recovery. Ann. Inst. Pasteur/Immunol. 136 D (1985) $57-65$.

11. Milich, D. R., Thornton, G. B., Neurath, A. R., Kent, S. B., Michel, M. L., Tiollais, P., Chisari, F. V.: Enhanced immunogenicity of the pre-S region of hepatitis B surface antigen. Science 228 (1985) 1195-1199. 